\title{
FAD-dependent oxidoreductase from Chaetomium thermophilum: Crystallographic fragment screening-based identification of putative substrates
}

\author{
L. Švecováa ${ }^{1,2}$, L. H. Østergaard ${ }^{3}$, T. Skálová ${ }^{1}$, T. Koval ${ }^{1}$, J. Dohnálek ${ }^{1}$ \\ ${ }^{I}$ Institute of Biotechnology of the Czech Academy of Sciences, v.v.i., Prümyslová 595, 25250 Vestec, Czech Republic, \\ ${ }^{2}$ Fac. of Nuclear Sciences and Physical Engineering, Czech Technical University in Prague, Břehová 7, 11519 Prague 1, Czech R. \\ ${ }^{3}$ Novozymes A/S, Biologiens Vej 2, 2800 Kgs. Lyngby, Denmark
}

\section{leona.svecova@ibt.cas.cz}

Novel FAD-dependent oxidoreductase from a lignocellulose-degrading fungus Chaetomium thermophilum (CtFDO) belongs to glucose-methanol-choline (GMC) superfamily of oxidoreductases which act on the hydroxyl groups of non-activated alcohols, carbohydrates and sterols. GMC superfamily enzymes share a two-domain character, the FAD-binding motif GxGxxG, and usually His-His or His-Asn active-site pair [1]. The crystal structure of $C t$ FDO reveals a unique His-Ser active-site pair a an active-site pocket, which is, compared to known structures of GMC oxidoreductases, unusually large, wide-open, and extended beyond the pyrimidine moiety of FAD. These features of the active-site pocket indicate a different type of substrate than common for GMC oxidoreductases.

A large activity screening with about 1000 compounds including substrates of GMC oxidoreductases showed $C t$ FDO to be inactive toward these compounds. To identify chemical groups of putative substrates and predict the substrates specificity of $C t$ FDO, we utilized the technique of crystallographic fragment screening, which resulted in series of six complexes binding small inorganic and aromatic moieties inside the active-site pocket of $C t$ FDO (Fig. 1). The size of the pocket together with preference for binding of aromatic moieties indicate polyaromatic nature of the putative substrate with molecular weight likely greater than $500 \mathrm{Da}$.

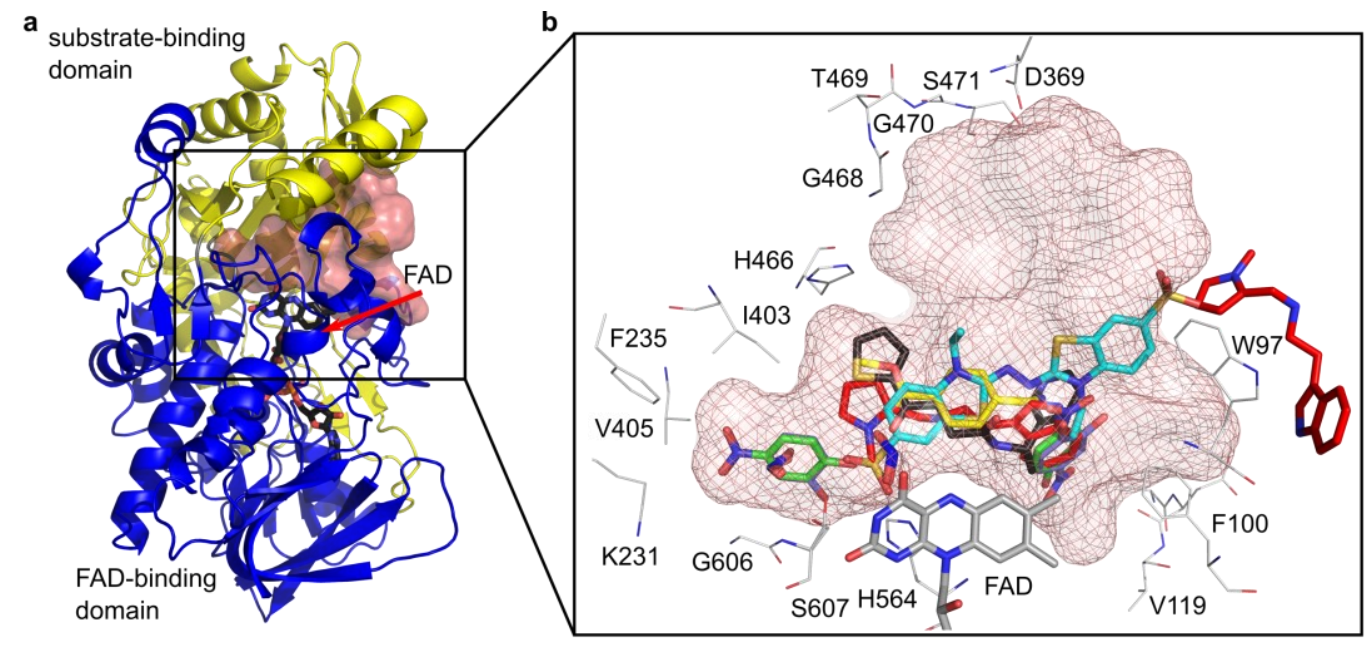

Figure 1. Crystal structure of CtFDO with color-coded substrate-binding (yellow) and the FAD-binding (blue) domains. The FAD cofactor is shown as sticks with black $\mathrm{C}$ atoms and the active-site pocket with salmon surface. (b) Three-dimensional superposition of the active sites of the CtFDO complexes binding four fragments from Frag Xtal Screen (Jena Bioscience) and two other compounds.

The active site pocket is displayed as salmon mesh, selected surrounding residues and FAD as sticks with gray $\mathrm{C}$ atoms, and the ligands with red, cyan, blank, yellow, green, purple C atoms. Tthe molecular graphics were created using PyMOL (Schrödinger).

[1] Sützl, L. Foley, G., Gillam, E. M. J., Bodén, M., Haltrich, D. (2019). Biotechnol Biofuels 12: 118.

[2] Švecová, L. Østergaard, L. H., Skálová, T., Schnorr, K., Koval’, T., Kolenko, P., Stránský, J., Sedlák, D., Dušková, J., Trundová, M., Hašek, J., Dohnálek, J. (2021). Acta Cryst. D77, 755-775.

Keywords: FAD-dependent oxidoreductase, glucose-methanol-choline superfamily of oxidoreductases, crystallographic fragment screening

The work was supported by the institutional support of IBT CAS, v.v.i. (RVO: 86652036), ERDF (CZ.02.1.01/0.0/0.0/15_003/0000447, CZ.02.1.01/0.0/0.0/16_013/0001776 and CZ.1.05/1.1.00/02.0109), MEYS CR (LM2018127 and CZ.02.1.01/0.0/0.0/16_019/0000778) and by the Grant Agency of the Czech Technical University in Prague (SGS19/189/OHK4/3T/14).

Acta Cryst. (2021), A77, C863 